\title{
Kidney Diseases
}

\section{Editors-in-Chief}

Zhi-Hong Liu - Nanjing University, Nanjing, China

\section{Consulting Editor}

Giuseppe Remuzzi - Mario Negri Institute for Pharmacological Research, Bergamo, Italy

\section{Associate Editors}

Chunsun Dai - Nanjing Medical University, Nanjing, China

Zheng Dong - Augusta University, Augusta, GA, USA

Chuan-Ming Hao - Fudan University, Shanghai, China

John Cijiang He - Icahn School of Medicine at Mount Sinai, New York, NY, USA

Gui-Sen Li - Sichuan Provincial People's Hospital, Sichuan, China

Jing Nie - Southern Medical University, Guangzhou, China

Jan Novak - University of Alabama at Birmingham, Birmingham, AL, USA

Fan Yi - Shandong University, Jinan, China

Shengqiang Yu - Second Military Medical University, Shanghai, China

Aihua Zhang - Nanjing Medical University, Nanjing, China

Hong Zhang - Peking University, Beijing, China

Jinghong Zhao - Third Military Medical University, Chongqing, China

\section{Editorial Board}

Ariela Benigni - Mario Negri Institute for Pharmacological Research, Bergamo, Italy Giovambattista Capasso - University of Campania "Luigi Vanvitelli", Naples, Italy Tak Mao Daniel Chan - The University of Hong Kong, Hong Kong, SAR, China Jianghua Chen - Zhejiang University, Hangzhou, China Nan Chen - Shanghai Jiao Tong University, Shanghai, China Alfred K. Cheung - University of Utah, Salt Lake City, UT, USA

Ricardo Correa-Rotter - National Medical Science and Nutrition Institute Salvador Zubiran,

Mexico City, Mexico

Kai-Uwe Eckardt - Charité - University Medicine Berlin, Berlin, Germany

Maik Gollasch - Charité - University Medicine Berlin, Berlin, Germany

Lynn Gomez - University of the Philippines, Bacoor, Philippines

Rujun Gong - University of Toledo College of Medicine, Toledo, OH, USA

Youfei Guan - Dalian Medical University, Dalian, China

Hai An Ha Phan - Hanoi Medical University, Hanoi, Vietnam

Berthold Hocher - University of Potsdam, Nuthetal, Germany

Fan-Fan Hou - Southern Medical University, Guangzhou, China

Vivekanand Jha - George Institute for Global Health, New Delhi, India

Duk-Hee Kang - Ewha Womans University, Seoul, Republic of Korea

Shin-Wook Kang - Yonsei University, Seoul, Republic of Korea 
Jeffrey B. Kopp - National Institute of Diabetes and Digestive and Kidney Diseases, Bethesda, MD, USA

Matthias Kretzler - University of Michigan, Ann Arbor, MI, USA

Hui Yao Lan - The Chinese University of Hong Kong, Hong Kong, SAR, China Philip K.T. Li - The Chinese University of Hong Kong, Hong Kong, SAR, China Sun Lin - Central South University, Changsha, China Bi-Cheng Liu - Southeast University, Nanjing, China Youhua Liu - University of Pittsburgh, Pittsburgh, PA, USA Toshio Miyata - Tohoku University, Sendai, Japan Masaomi Nangaku - The University of Tokyo, Tokyo, Japan Vlado Perkovic - George University, Newtown, NSW, Australia Qi Qian - Mayo Clinic College of Medicine, Rochester, MN, USA Michael Rocco - Wake Forest University, Winston-Salem, NC, USA Claudio Ronco - St. Bartolo Hospital, Vicenza, Italy Pierre Ronco - UPMC University Pierre and Marie Curie Paris 6, Paris, France Xiong Z. Ruan - University College London, London, UK Ivan Rychlik - Charles University, Prague, Czech Republic Bancha Satirapoj - Phramongkutklao Hospital, Bangkok, Thailand Stuart J. Shankland - University of Washington, Seattle, WA, USA Shaolin Shi - Nanjing University, Nanjing, China Yusuke Suzuki - Juntendo University, Tokyo, Japan Charles Swanepoel - University of Cape Town, Cape Town, South Africa Cheuk Chun Szeto - The Chinese University of Hong Kong, Hong Kong, SAR, China Sydney Tang - The University of Hong Kong, Hong Kong, SAR, China Vladimir Tesar - Charles University, Prague, Czech Republic Yasuhiko Tomino - Medical Corporation Showakai, Tokyo, Japan Marcello Tonelli - University of Calgary, Calgary, AB, Canada

Hernán Trimarchi - British Hospital Buenos Aires, Buenos Aires, Argentina Raymond Vanholder - Ghent University Hospital - UZ Ghent, Ghent, Belgium Christoph Wanner - University Hospital of Würzburg, Würzburg, Germany Qihe Xu - King's College London, London, UK Chih-Wei Yang - Chang Gung Memorial Hospital, Taipei, Taiwan, ROC Tianxin Yang - University of Utah, Salt Lake City, UT, USA Lianghong Yin - Jinan University, Guangzhou, China Xueqing Yu - Sun Yat-Sen University, Guangzhou, China Caihong Zeng - Jinling Hospital, Nanjing, China Ming-hui Zhao - Peking University, Beijing, China Xiaoai Zhao - Stanford University, Stanford, CA, USA Haitao Zhang - Nanjing University, Nanjing, China Shougang Zhuang - Rhode Island Hospital, Providence, RI, USA 
S. Karger

Medical and Scientific Publishers

Basel $\cdot$ Freiburg $\cdot$ Hartford $•$ Oxford $•$ Bangkok $\cdot$ Dubai $\cdot$ Kuala Lumpur $•$ Melbourne $\cdot$ Mexico City $\cdot$ Moscow $•$ New Delhi $\bullet$ Paris $\bullet$ Shanghai $\cdot$ Tokyo

\section{Disclaimer}

The statements, opinions and data contained in this publication are solely those of the individual authors and contributors and not of the publisher and the editor(s). The appearance of advertisements in the journal is not a warranty, endorsement, or approval of the products or services advertised or of their effectiveness, quality or safety. The publisher and the editor(s) disclaim responsibility for any injury to persons or property resulting from any ideas, methods, instructions or products referred to in the content or advertisements.

Drug Dosage

The authors and the publisher have exerted every effort to en sure that drug selection and dosage set forth in this text are in accord with current recommendations and practice at the time of publication. However, in view of ongoing research, changes in government regulations, and the constant flow of information relating to drug therapy and drug reactions, the reader is urged to check the package insert for each drug for any change in indications and dosage and for added warnings and precautions. This is particularly important when the recommended agent is a new and/or infrequently employed drug.
All rights reserved.

No part of this publication may be translated into other languages, reproduced or utilized in any form or by any means, electronic or mechanical, including photocopying, recording, microcopying, or by any information storage and retrieval system, without permission in writing from the publisher or in the case of photocopying, direct payment of a specified fee to the Copyright Clearance Center (see "General Information")

(c) Copyright 2021 by S. Karger AG

CH-4009 Basel (Switzerland)

Printed on acid-free and non-aging paper (ISO 9706) 


\section{No. 1}

\section{Review Articles}

1 Hypoxia-Inducible Factor-Proline Hydroxylase Inhibitor in the Treatment of Renal Anemia

Hu, X.; Xie, J.; Chen, N. (Shanghai)

10 Pediatric Acute Kidney Injury to the Subsequent CKD Transition

Wang, F.; Ding, J. (Beijing)

14 Toll-Like Receptors Regulate the Development and Progression of Renal Diseases Liu, M.; Zen, K. (Nanjing)

Systematic Review and Meta-Analysis

24 Prognostic Value of Serum Magnesium in Mortality Risk among Patients on Hemodialysis: A Meta-Analysis of Observational Studies

Wu, H. (Guangzhou); Li, Q. (Dongguan); Fan, L.; Zeng, D. (Guangzhou); Chi, X. (Zhongshan/Mannheim); Guan, B.; Hu, B. (Guangzhou); Lu, Y. (Guangzhou/Mannheim); Yun, C.; Krämer, B. (Mannheim); Hocher, B. (Guangzhou/Mannheim); Liu, F.; Yin, L. (Guangzhou)

Research Articles

34 Synergistic Inhibition of Renal Fibrosis by Nintedanib and Gefitinib in a Murine Model of Obstructive Nephropathy Feng, L.; Li, W.; Chao, Y.; Huan, Q.; Lu, F.; Yi, W.; Jun, W.; Binbin, C.; Na, L. (Shanghai); Shougang, Z. (Shanghai/Providence, RI)

50 Severe Infections following Rituximab Treatment in Antineutrophil Cytoplasmic Antibody-Associated Vasculitis Li, Z.-Y.; Chen, M.; Zhao, M.-H. (Beijing)

57 Infectious Complications in Patients with Primary Glomerulonephritis over 10 Years: A Single-Center Experience in Turkey

Eren Sadioglu, R.; Eyupoglu, S.; Erdogmus, S.; Kumru Sahin, G.; Yoruk, F.; Kutlay, S.; Keven, K.; Erturk, S.; Sengul, S. (Ankara)

67 Severity of Intrarenal Arterial Lesions Can Predict the Clinical Prognosis of Hepatitis B Virus-Associated Glomerulonephritis: A Retrospective Study Zhuang, Y.; Liu, B.; Yu, Y. (Fuzhou); Guan, T. (Xiamen); Zheng, Z. (Fuzhou); Chen, A. (Xiamen)

\section{No. 2}

Review Articles

79 An Update for Mesenchymal Stem Cell Therapy in Lupus Nephritis

Li, W.; Chen, W.; Sun, L. (Nanjing)
90 Periprocedural Peritonitis Prophylaxis: A Summary of the Microbiology and the Role of Systemic Antimicrobials Hsueh, L.; Hu, S.L.; Shah, A.D. (Providence, RI)

Systematic Review and Meta-Analysis

100 Renal Injury by SARS-CoV-2 Infection: A Systematic Review Wang, M.; Xiong, H.; Chen, H.; Li, Q. (Chongqing); Ruan, X.Z. (Chongqing/ London)

Research Articles

111 Risk Factors and Outcomes of Acute Kidney Injury in Critically III Patients with Coronavirus Disease 2019

Cheng, Y.; Zhang, N.; Luo, R.; Zhang, M.; Wang, Z.; Dong, L.; Li, J.; Zeng, R.; Yao, Y.; Ge, S.; Xu, G. (Wuhan)

120 Incorporation of Urinary Neutrophil Gelatinase-Associated Lipocalin and Computed Tomography Quantification to Predict Acute Kidney Injury and In-Hospital Death in COVID-19 Patients

He, L.; Zhang, Q.; Li, Z.; Shen, L.; Zhang, J.; Wang, P.; Wu, S.; Zhou, T.; Xu, Q.; Chen, X.; Fan, X.; Fan, Y.; Wang, N. (Shanghai)

131 Evaluation of Renal Fibrosis by Mapping Histology and Magnetic Resonance Imaging

Zhang, J.; Yu, Y. (Nanjing); Liu, X. (Beijing); Tang, X.; Xu, F.; Zhang, M. (Nanjing); Xie, G. (Beijing); Zhang, L. (Nanjing); Li, X. (Beijing); Liu, Z.-H. (Nanjing)

143 Drug-Induced Hospital-Acquired Acute Kidney Injury in China: A Multicenter Cross-Sectional Survey

Liu, C.; Yan, S.; Wang, Y.; Wang, J. (Beijing); Fu, X. (Changchun); Song, H. (Fuzhou); Tong, R. (Chengdu); Dong, M. (Harbin); Ge, W. (Nanjing); Wang, J. (Beijing); Yang, H. (Xining); Wang, C. (Fuzhou); Xia, P. (Chongqing); Zhao, L. (Shenyang); Shen, S. (Beijing); Xie, J. (Guiyang); Xu, Y. (Tianjin); Ma, P. (Zhengzhou); Li, H. (Jinan); Lu, S. (Nanchang); Ding, Y. (Wuhan); Jiang, L. (Hefei); Lin, Y. (Beijing); Wang, M. (Xi'an); Qiu, F. (Chongqing); Feng, W.; Yang, L. (Beijing)

156 Unveiling the Features of Mercury-Associated Minimal Change Disease: Comparison with Primary Minimal Change Disease Qin, A.; Yu, X.; Wang, S.; Zhou, F.; Zhao, M. (Beijing)

No. 3

Review Articles

167 Tackling Dialysis Burden around the World: A Global Challenge

Li, P.K.-T.; Chan, G.C.-K. (Shatin); Chen, J. (Hangzhou); Chen, H.-C (Kaohsiung); Cheng, Y.-L. (Tai Po); Fan, S.L.-S. (London); He, J.C. (New York, NY); Hu, W. (Nanjing); Lim, W.-H. (Perth, WA); Pei, Y (Toronto, ON); Teo, B.W. (Singapore); Zhang, P. (Hangzhou); Yu, X. (Guangzhou); Liu, Z.-H. (Nanjing)

176 The Role of CXCL12 in Kidney Diseases: A Friend or Foe? Song, A.; Jiang, A.; Xiong, W.; Zhang, C. (Wuhan) karger@karger.com

www.karger.com

Karger
(C) 2021 S. Karger AG, Base

Access to full text and tables of contents, including tentative ones for forthcoming issues: www.karger.com/kdd_issues 
Research Articles

186 Nicotinamide Supplementation Attenuates Renal Interstitial Fibrosis via Boosting the Activity of Sirtuins

Zhen, X.; Zhang, S.; Xie, F.; Zhou, M.; Hu, Z.; Zhu, F.; Nie, J. (Guangzhou)

200 Monozygotic Twins Discordant for Immunoglobulin A Nephropathy Display Differences in DNA Methylation and Gene Expression

Wei, M.; Meng, S.; Shi, S.; Liu, L.; Zhou, X.; Lv, J.; Zhu, L.; Zhang, H. (Beijing)

210 Hyperuricemia and Impaired Renal Function: A Prospective Cohort Study

Aiumtrakul, N.; Wiputhanuphongs, P.; Supasyndh, O.; Satirapoj, B. (Bangkok)

219 Lower Serum Irisin Levels Are Associated with Increased Abdominal Aortic Calcification in Peritoneal Dialysis Patients Zhou, S.-J.; Wang, X.-X.; Tang, W.; Han, Q.-F.; He, L.; Zhang, A.-H. (Beijing)

227 Fibroblast Growth Factor 21 Predicts and Promotes Vascular Calcification in Haemodialysis Patients

Jiang, L. (Nanjing/Suzhou); Yin, Q. (Nanjing); Yang, M.; Li, M. (Changzhou); Pan, M.; Han, Y.; Zhao, Z.; Wang, Z.; Zhu, L.; Wei, Q.; Tu, Y.; Gao, M.; Liu, H.; Zhang, X.; Liu, B.; Wang, B. (Nanjing)

Letter to the Editor

241 The Neutrophil to Lymphocyte Ratio: An Ideal Marker for Early Diagnosis and Short-Term Prognosis of Acute Kidney Injury?

Lang, S.M. (Jena); Schiffl, H. (Munich)

Reply

244 Reply to "The Neutrophil-to-Lymphocyte Ratio: An Ideal Marker for Early Diagnosis and Short-Term Prognosis of Acute Kidney Injury?"

Zhu, J. (Changsha); Dong, Z. (Changsha/Augusta, GA)

No. 4

World Kidney Day 2021: Editorial

247 Living Well with Kidney Disease by Patient and Care-Partner Empowerment: Kidney Health for Everyone Everywhere Kalantar-Zadeh, K. (Orange, CA); Li, P.K.-T. (Hong Kong); Tantisattamo, E. (Orange, CA); Kumaraswami, L. (Chennai); Liakopoulos, V. (Thessaloniki); Lui, S.-F. (Hong Kong); Ulasi, I. (Enugu); Andreoli, S. (Indianapolis, IN); Balducci, A. (Rome); Dupuis, S. (Brussels); Harris, T. (London); Hradsky, A. (Brussels); Knight, R. (Tampa, FL); Kumar, S. (Chennai); Ng, M. (Hong Kong); Poidevin, A. (Brussels); Saadi, G (Giza); Tong, A. (Sydney, NSW); for the World Kidney Day Steering Committee

Review Articles

254 Lysosome Depletion-Triggered Autophagy Impairment in Progressive Kidney Injury

Chen, X.; Li, Z.; Yang, C.; Tang, J. (Zhanjiang); Lan, H. (Hong Kong SAR); Liu, H. (Zhanjiang)

268 Bone Quality in CKD Patients: Current Concepts and Future Directions - Part I

Asadipooya, K. (Lexington, KY); Abdalbary, M. (Lexington, KY/

Mansoura); Ahmad, Y.; Kakani, E.; Monier-Faugere, M.-C.; El-Husseini, A. (Lexington, $\mathrm{KY}$ )
Research Articles

278 Penicilliumin B Protects against Cisplatin-Induced Renal Tubular Cell Apoptosis through Activation of AMPK-Induced Autophagy and Mitochondrial Biogenesis

Shen, W.; Jia, N.; Miao, J.; Chen, S.; Zhou, S.; Meng, P.; Zhou, X.; Tang, L.; Zhou, L. (Guangzhou)

293 SIRT1 Alleviates Aldosterone-Induced Podocyte Injury by Suppressing Mitochondrial Dysfunction and NLRP3 Inflammasome Activation Jiang, M.; Zhao, M.; Bai, M.; Lei, J.; Yuan, Y.; Huang, S.; Zhang, Y.; Ding, G.; Jia, Z.; Zhang, A. (Nanjing)

306 A Novel Standardized Method of Renal Biopsy in Mice Mao, X. (Harbin); Wang, C. (Harbin/Haikou); Xu, Z.; He, Y.; Hou, Y. (Harbin); Li, B. (Harbin/Haikou)

315 Physical Function and Clinical Outcomes in Hemodialysis Patients: China Dialysis Outcomes and Practice Patterns Study

Niu, Q.; Zhao, X.; Gan, L. (Beijing); Liang, X. (Guangzhou); Ni, Z.; Chen, X (Shanghai); Chen, Y. (Beijing); Hou, F.F. (Guangzhou); Zuo, L. (Beijing)

323 Effect of Peer Mentoring on Quality of Life among CKD Patients: Randomized Controlled Trial

Ghahramani, N.; Chinchilli, V.M.; Kraschnewski, J.L.; Lengerich, E.J.; Sciamanna, C.N. (Hershey, PA)

No. 5

Review Articles

335 Studying Kidney Diseases at the Single-Cell Level Jiang, M.; Chen, H.; Guo, G. (Hangzhou)

343 Tolvaptan in Pediatric Autosomal Dominant Polycystic Kidney Disease: From Here to Where?

Liu, F; Feng, C.; Shen, H.; Fu, H.; Mao, J. (Hangzhou)

350 Mechanisms of Scarring in Focal Segmental Glomerulosclerosis Sun, K.; Xie, Q. (Shanghai); Hao, C.-M. (Shanghai/Nashville, TN)

359 Bone Quality in Chronic Kidney Disease Patients: Current Concepts and Future Directions - Part II Asadipooya, K. (Lexington, KY); Abdalbary, M. (Lexington, KY/ Mansoura); Ahmad, Y.; Kakani, E.; Monier-Faugere, M.-C.; El-Husseini, A. (Lexington, KY)

Research Articles

372 Smad3-Targeted Therapy Protects against Cisplatin-Induced AKI by Attenuating Programmed Cell Death and Inflammation via a NOX4-Dependent Mechanism Yang, Q.; Gao, L.; Hu, X.; Wang, J.; Zhang, Y.; Dong, Y. (Hefei); Lan, H.Y. (Hong Kong); Meng, X. (Hefei)

391 Effect of Marital Status on Depression and Mortality among Patients with Chronic Kidney Disease from National Health and Nutrition Examination Survey 2005-2014 Wang, Z.; Cheng, Y.; Zhang, N.-H.; Luo, R.; Guo, K.; Ge, S.-W.; Xu, G. (Wuhan)

401 Hydroxychloroquine Inhibits Cardiac Conduction in Aged Patients with Nonmalaria Diseases

Yu, Y.; Xu, J.; Xie, A.; Liu, S.; Wang, X.; Zhu, R.; Wang, X. (Nanjing) 
Systematic Review and Meta-Analysis

411 Blood Pressure Variability and Prognosis in Hemodialysis Patients: A Systematic Review and Meta-Analysis

Zhao, Y.; Yang, L.; Yu, S. (Chengdu); Salerno, S.; Li, Y. (Ann Arbor, MI); Cui, T.; Zhang, L.; Fu, P. (Chengdu)

\section{No. 6}

Review Articles

425 Next-Generation Sequencing-Based Genetic Diagnostic Strategies of Inherited Kidney Diseases

Zhang, J. (Hangzhou); Zhang, C. (Nanjing/Hangzhou); Gao, E. (Nanjing); Zhou, Q. (Hangzhou)

438 The Updates of Podocyte Lipid Metabolism in Proteinuric Kidney Disease

Sun, Y.; Cui, S.; Hou, Y.; Yi, F. (Jinan)

452 Metabolic Acidosis in Patients with CKD: Epidemiology, Pathogenesis, and Treatment

Adamczak, M.; Surma, S. (Katowice)

468 Endostatin in Renal and Cardiovascular Diseases

Li, M.; Popovic, Z. (Heidelberg); Chu, C. (Heidelberg/Berlin); Krämer, B.K. (Heidelberg/Mannheim); Hocher, B. (Heidelberg/Changsha/Berlin)
Research Articles

482 Intrauterine Low-Protein Diet Exacerbates Abnormal Development of the Urinary System in Gen 1-Mutant Mice Yu, M.; Li, Y.; Tan, L.; Chen, J.; Zhai, Y.; Rao, J.; Fang, X.; Liu, J.; Liu, J.; Wu, X.; Xu, H.; Shen, Q. (Shanghai)

494 A Phase 3 Study of Enarodustat (JTZ-951) in Japanese Hemodialysis Patients for Treatment of Anemia in Chronic Kidney Disease: SYMPHONY HD Study

Akizawa, T.; Nangaku, M. (Tokyo); Yamaguchi, T. (Miyagi); Koretomo, R.; Maeda, K.; Miyazawa, Y. (Tokyo); Hirakata, H. (Fukuoka)

503 Serum Sortilin Is Associated with Coronary Artery Calcification and Cardiovascular and Cerebrovascular Events in Maintenance Hemodialysis Patients

$\mathrm{Xu}$, J. (Changsha); Shen, C.-J. (Zhuzhou); Ooi, J.D. (Clayton, VIC);

Tang, Y.-S.; Xiao, Z.; Yuan, Q.-J.; Zhong, Y.; Zhou, Q.-L. (Changsha)

Case Report

514 Preimplantation Genetic Testing Prevented Intergenerational Transmission of X-Linked Alport Syndrome

Hu, X.; Zhang, J.; Lv, Y.; Chen, X.; Feng, G.; Wang, L.; Ye, Y.; Jin, F.; Zhu, Y. (Hangzhou)

521 Acknowledgement to the Reviewers 